\title{
Thermographic non destructive control of joint of ceramic matrix composite tubes
}

\author{
By F. Cernuschi, L. Lorenzoni \\ CESI, Via Reggio Emilia, 3920090 Segrate (MI) Italia, E-mail: cernuschi@cesi.it
}

\begin{abstract}
A thermographic technique for the assessment of the quality of joints of ceramic matrix composite tubes is presented: this method is based on the time resolved analysis of the surface temperature rise when a stepwise heating is produced within the pipe. Starting from experimental data, a figure of merit for joint thermal resistance was defined and successfully applied to jointed pipes, to a single pipe (perfect joint) and to a pair of pipes inserted one inside the other one but without any joining material (worst joint).
\end{abstract}

\section{Introduction}

In the frame of the Brite-Euram Project BE 3089/UHTHE/EFCC/B4, devoted to the development and application of design and integration technologies for industrial sub-critical components based on CMC-materials, ceramic-ceramic permanent joint methodologies have been developed. In particular joining has been applied to tubular components for Ultra High Temperature Heat Exchanger (UHTHE).

The assessment of the joining process repeatability as well as the uniformity of the joining material distribution in the joint area is a key point as far as the reliability of the component is concerned.

As fibre reinforced materials scatter significantly ultrasonic waves, other non destructive techniques have been investigated; in principle an attractive method is represented by active infrared thermography because it is contactless and allows to image wide areas and its application is not restricted to laboratory.

The joint geometry is conical; this means that the two tubular parts are manufactured as shown in Fig. 1. After joining the overall thickness of joint area should be roughly the same as elsewhere along the pipe.

The idea the developed methodology is based on, consists in estimating the thermal resistance of joints; in fact the higher the thermal resistance the poorer the joint as air is surely less conductive than any joining material.

A first attempt was made by completely inserting within the pipe a linear lamp as heating source and looking for hot or cold spots in the joint area but it resulted to be practically impossible to distinguish between joint defects and other features of the component due to the conical joint geometry and to the CMC material non uniformity. In particular close to the joint the thickness could be not regular as supposed and in any case the manufacturing process of CMC components can not guarantee a strong uniformity of the material density and consequently of thermal properties.

Although the effects of these features of the components can not be completely overcome a time resolved analysis of the temperature rise when a stepwise heating is produced within the pipe could provide more significant information as far as the joint control is concerned. 


\section{Experimental}

\subsection{The experimental set-up}

The infrared camera used for this experimental activity is a Focal Plane Array (320x240pixel) CEDIP Jade system sensitive in the spectral range $8-10 \mu \mathrm{m}$ with a NETD of $30 \mathrm{mK}$ and able to acquire snapshot infrared images with a frequency rate ranging from 50 up to $1000 \mathrm{~Hz}$ as a function of the image dimension.

The heating source was a $900 \mathrm{~W}$ linear lamp with a length of about $400 \mathrm{~mm}$. The time duration of the heating was fixed at $1.5 \mathrm{~s}$ by using a timer connected to the power supplier.

In order to hold and rotate around its axis the pipe a cylindrical holder fixed to a rotation stage was used as shown in Fig. 2.

\subsection{The technique: a qualitative approach}

In order to estimate the thermal resistance of jointed pipes by time resolved infrared thermography, a linear lamp was inserted within the pipe along its axis with one end positioned just in correspondence of the beginning of the overlapping region between the two tubular parts (see Fig. 2). In this way, when the lamp is switched on heat diffusion within the joint takes place both radially and axially but depending from the thermal resistance of the joint itself the spatial distribution as well as the time evolution of the surface temperature in this region can differ also significantly.

In fact in the case of a poor joint, heat diffuses mainly axially along the conical insertion while a good joint allows the heat to remarkably diffuse also in the radial direction.

Experimentally in order to compare the effects of a different thermal resistance on the surface temperature for jointed pipes, for ideal and worst joints, we have assumed as ideal joint a portion of the pipe far away from the joint (as a matter of fact bulk material should exhibit the lowest thermal resistance) and as worst joint the one without any joining material corresponding to insert just the two pipes one into the each other without filling the small gap between them.

Comparing experimentally the surface temperature for the three different cases the results are the following: in the first case the spatial distribution appears nearly uniform in the joint part (not directly heated by the lamp) as shown in Fig.3a; on the contrary in the cases of a good and ideal joints the surface temperature strongly decreases moving away from the heating source (see Fig. 3b).

Analogously the time evolution of the surface temperature in different positions taken along a line parallel to the pipe axis shows complete different trends as a function of the thermal resistance of the joint.

Fig. $4 a-4 c$ show the surface temperature as a function of time during heating in 8 small areas as indicated by Fig. 5 .

These results furnish useful indications about the possibility of discriminating between good and poor joints in terms of thermal resistance by using an infrared camera but the development of a specific algorithm is required in order to estimate the reproducibility of the joining process and thus to rank the joints.

\subsection{The technique: a quantitative approach}

Looking at Fig.5a-5c it appears evident that both the shape and the maximum of the temperature versus time curves for the areas on the left side of the pipe (those not directly heated) are strongly related to the thermal resistance of the joints. On the contrary shape and maximum of curves related to those areas directly heated by the lamp are obviously independent from the joint quality. In order to reduce as much as possible the influence of experimental factors like for example heating amplitude variations, data referring to areas on the joint should be normalised in respect of data obtained from these transition curves. 
In particular we propose to obtain as representative parameters the transition height and the transition centre of the temperature vs. time curves referring both to areas over the joint and away from it. For these to types of curves the best analytical representations seem to be the Reverse Asymmetric Sigmoid and the Asymmetric Sigmoid respectively. The equations describing these to functions families are:

$$
y=a+b\left[1-\frac{1}{\left\{1+\exp \left(-\frac{\left.x-d \ln \left(2^{1 / e}-1\right)-c\right)}{d}\right)\right\}^{e}}\right]
$$

where $a, b, c, d, e$ are fitting parameters; in particular the transition height and the transition centre in both cases correspond to $b$ and $c$. Thus a possible figure of merit of joints could be defined (for a fixed area over the joint) as the ratio $b / c$ normalised by the same ratio but referred to a fixed area away from the joint and directly heated be the lamp (the right side of the pipe as shown in Fig. 5). All the parameters needed for this computation can be obtained by fitting experimental data with eq.(1) and eq. (2) respectively as shown in Fig. $6 \mathrm{a}$ and $6 \mathrm{~b}$ for areas 3 and 7 of Fig 5. Fig. $6 \mathrm{~b}$ shows also graphically the meaning of these two parameters for an asymmetric sigmoid ( for the reverse asymmetric sigmoid the meaning of the two parameters is complete similar)and Table 1 furnishes the results obtained by applying this procedure to the experimental curves of temperature vs. time for the areas 3 and 7 (see Fig. 5) for six jointed pipes.

The reproducibility of the methodology was estimated by repeating the measurement either without moving anything or repositioning pipe and lamp. The standard deviation of the mean value resulted \pm 0.008 and \pm 0.012 respectively. Measurements were carried out at three different angular position but variations resulted of the same order of experimental uncertainty.

\section{Conclusion}

Values of the figure of merit obtained applying the proposed algorithm to experimental data of six jointed pipes, of one not jointed pipe and of an ideally jointed pipe all give evidence of the capability of the technique in ranking joints. In any case in order to confirm these preliminary results a comparison is required between these values and the values of tensile tests which will be carried out on these jointed pipes.

Table 1 Figure of merit for some jointed pipes

\begin{tabular}{c|c|c|c|c|c|c|c} 
Perfect joint & False Joint & Joint 1 & Joint 2 & Joint 3 & Joint 4 & Joint 5 & Joint 6 \\
\hline $\begin{array}{c}0.34 \\
\text { Acknowledgements }\end{array}$ & 0.1 & 0.24 & 0.29 & 0.28 & 0.26 & 0.29 & 0.32 \\
\end{tabular}


The authors would like to thank the partners of the Brite Project EFCC/UHTHE/B4 especially dr. R. Weiss and Mr. M. Heinrich of Schunk Kohlenstofftechnik GmbH Giessen Germany, for helpful discussions and the accurate and on time supply of the samples.

Fig. 1 A sketch of the conical joint.

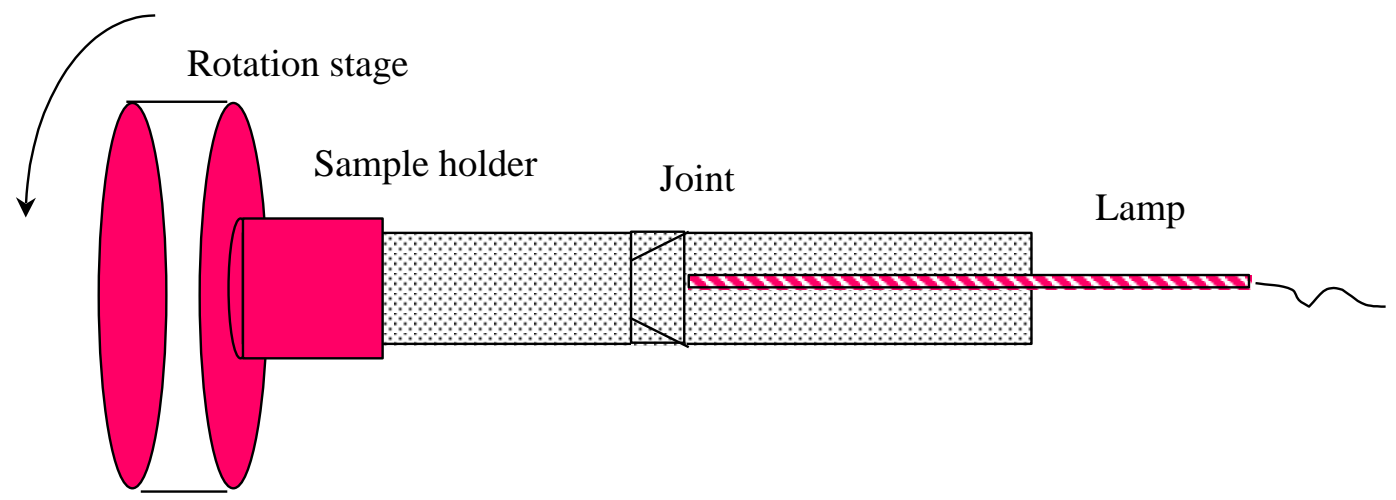

Fig. 2 A sketch of the sample holder

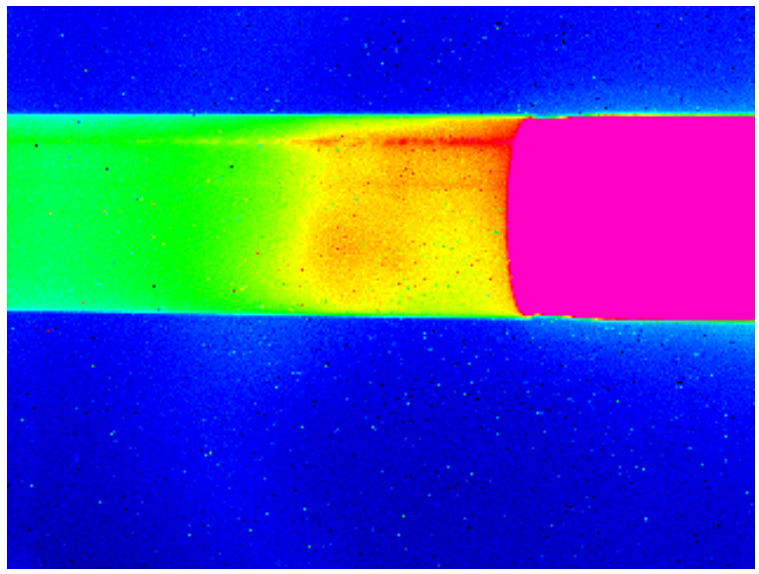

(a)

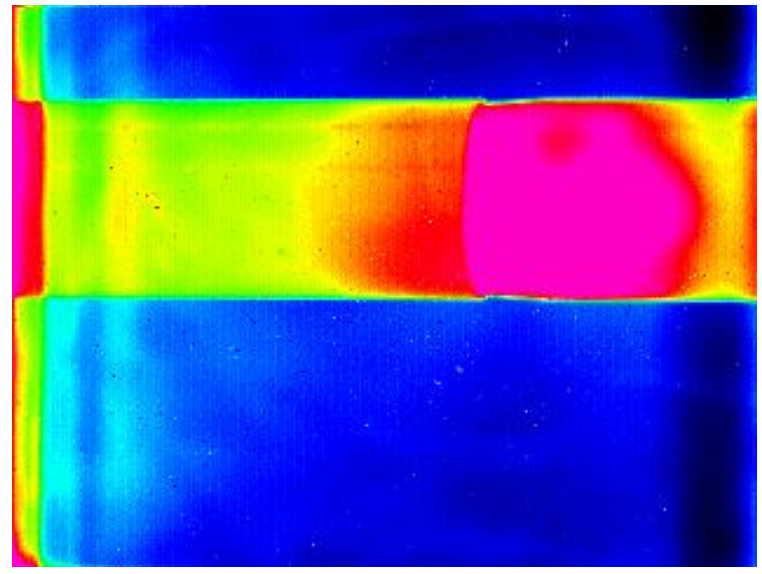

(b)

Fig. 3 Infrared images of (a) the non jointed pipe and of (b) a jointed pipe.

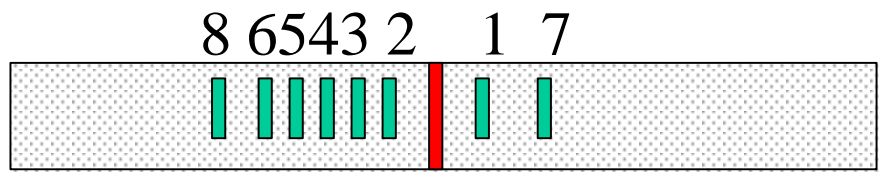

Fig. 5 Position of the areas where the mean value of the temperature vs. time is considered in time resolved experiments. 
http://dx.doi.org/10.21611/qirt.2000.012

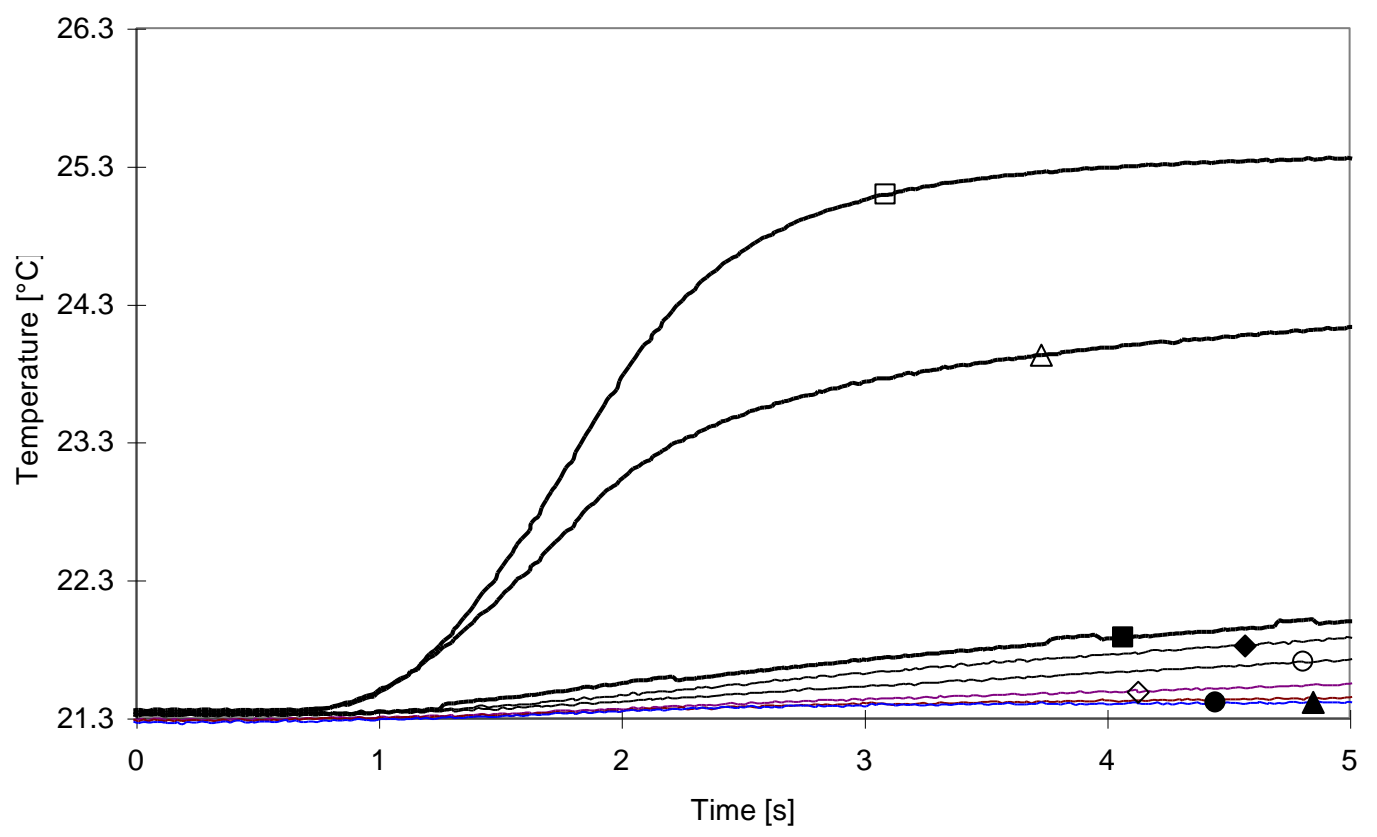

(a)

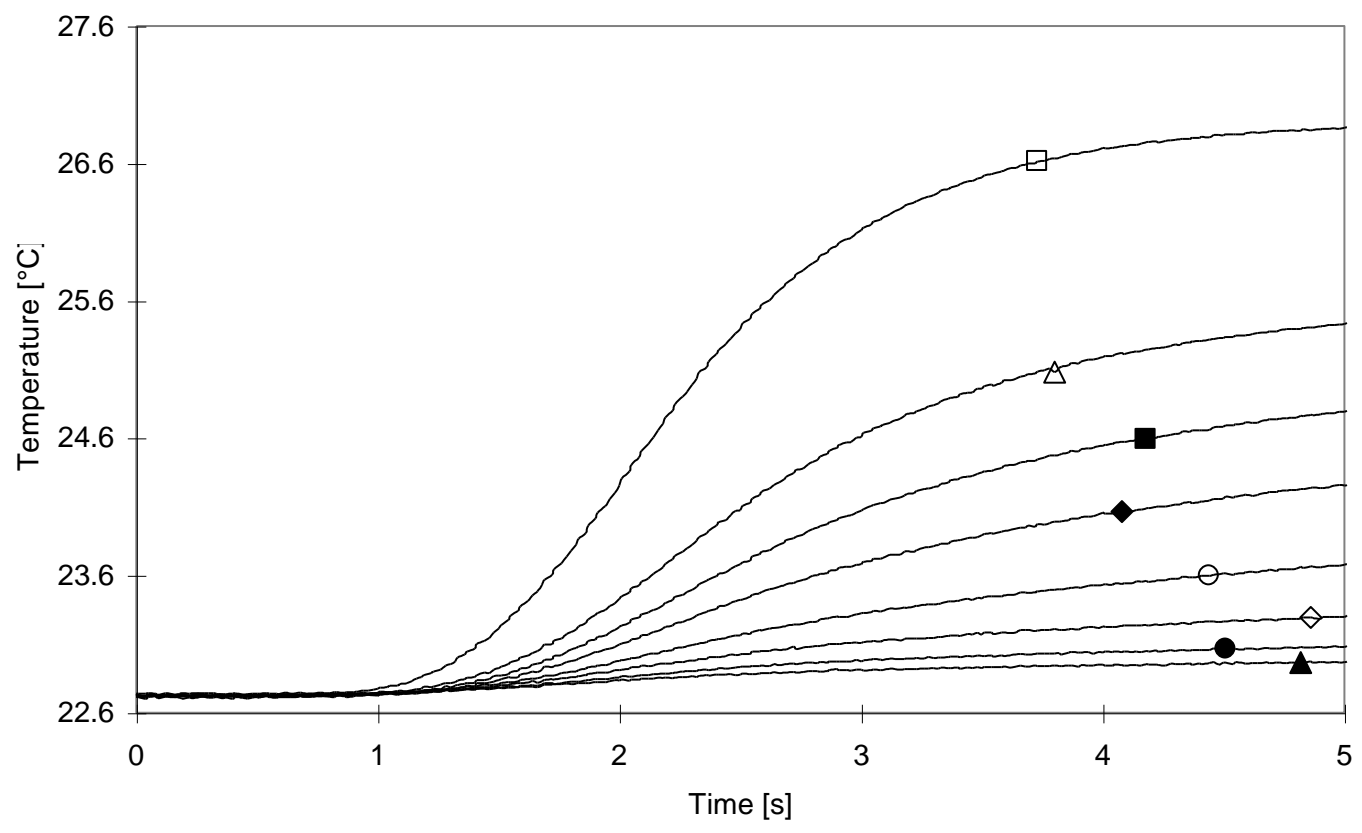

(b) 


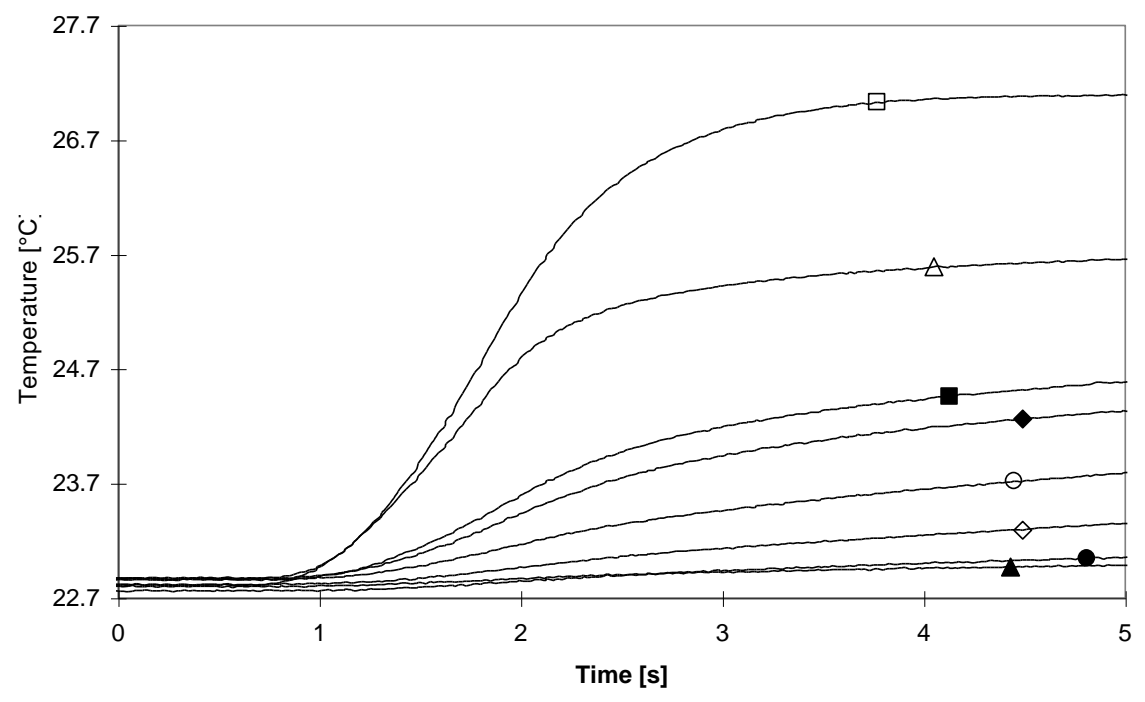

(c)

Fig. 4 Temperature vs. time at 8 different locations as shown in Fig. 5 for (a) the worst jointed pipe, (b) the ideal jointed pipe and (c) a jointed pipe. The correspondence between positions and symbols is the following: $(\Delta)$ point $1,(\square)$ point $2,(\diamond)$ point $3,(\bigcirc)$ point $4,(\diamond)$ point $5,(\bullet)$ point $6,(\square)$ point 7 and $(\boldsymbol{\Delta})$ point 8.

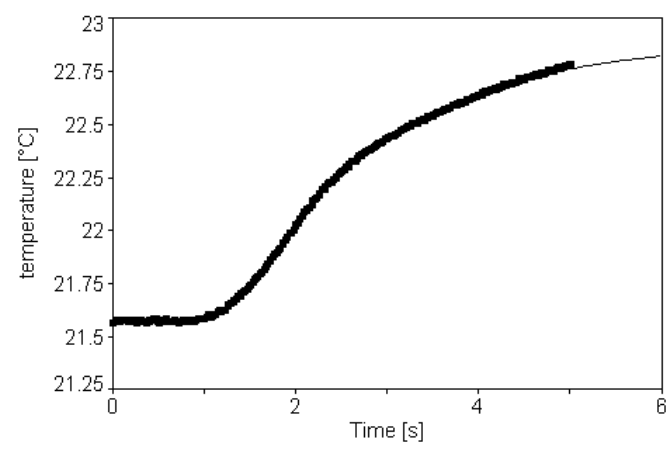

(a)

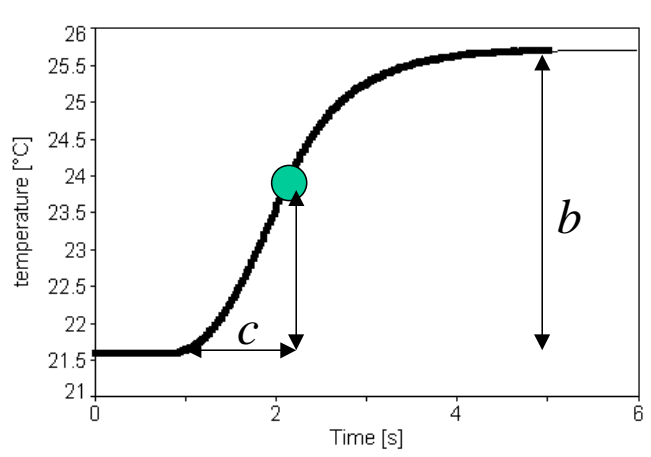

(b)

Fig. 6 Experimental data of (a) area 3 fitted by an reverse asymmetric sigmoid and of (b) area 7 fitted by an asymmetric sigmoid. Data refer to the pipe $n^{\circ} 4$. The meaning of the parameters $b$ and $c$ is graphically shown in (b) as well. 